\title{
Surveying the Development of Authorship Identification of Text Messages
}

\author{
Abdulaziz Altamimi ${ }^{1}$, Saud Alotaibi ${ }^{1}$ and Abdulrahman Alruban ${ }^{1,2}$ \\ ${ }^{1}$ Centre for Security, Communications and Network Research, \\ Plymouth University, Plymouth, UK \\ ${ }^{2}$ Computer Sciences and Information Technology College, Majmaah University, \\ Al Majma'ah, Saudi Arabia
}

\begin{abstract}
People typically use multiple messaging systems and send text messages concurrently by different messaging systems such as SMS, email, Twitter, and Facebook. This means that these forms of messaging systems are highly integrated into people's everyday activities. Moreover, forensic science has had many challenges in many different kinds of crimes, differing from physical crimes to computer mediated crime activities. Identification of suspects has become crucial for law enforcement due in particularly to the anonymity that the internet and associated services provide and identify the ownership of messages. In this paper, survey the development of existing author identification have been done by establishing a comprehensive review of author identification to determining the core approaches and techniques utilized. Furthermore, this study also examines possible challenges in author identification and points to some of the open problems which need to be tackled.
\end{abstract}

\section{Introduction}

The increasing popularity and diversity of messaging systems today such as WhatsApp, SMS, Facebook, Twitter, Email, WeChat, and LinkedIn, has enabled people to communicate with each other in a convenient way via their mobile devices or Internet connected computers [1]. Globally, there are 50 billion messages (text, tweets, instant message posts) sent every day, that is, more than 18 trillion over 12 months across a variety of platforms [2]. Figure 1 illustrates the monthly active users on several popular social networks and messaging systems around the world during 2011-2015, demonstrating an increasing trend of usage across these messaging systems. For example, monthly active users of WhatsApp increased from 700 million in 2014 to 1 billion in 2015 .

However, despite the popularity of messaging systems, they are often found to be the source and target for criminal activities. Messaging systems has become an ideal place for criminals due to its characteristics such as anonymity [3, 4], ease of use and low of cost [5]. This leads to a variety of direct and indirect criminal activities such as, sending spam texts to gain personal information [6], grooming children, kidnap, murder, terrorism and violence $[7,8]$.

A need exists therefore to be able to identify the ownership of messages sent across these systems. Unfortunately, relying on just the account details to simply identify the author of that account could be misleading because messaging platforms typically do not enforce identity checking, enabling the creation of fake accounts or accounts which are not easily traced back to an individual [9]. Authorship identification is however an approach that provides the ability to determine the authenticity of the author through an examination of the messages.

The remainder of this paper is organized as follows. Section 2 presents author identification concept with a detailed explanation of the research methodology for this systematic literature review. A literature review of author identification is given in section 3 and section 4 presents the results and discussion. Section 5 discusses existing research solutions in detail and highlights open issues. Finally, section 6 highlights the conclusions of this paper.

\section{Author Identification}

Forensic science has had many challenges in many different kinds of crimes, differing from physical crimes to computer mediated crime activities. Identification of suspects has become crucial for law enforcement due in particularly to the anonymity that the internet and associated services provide. Author identification (authorship attribution) is deciding the likelihood of a piece of writing to be produced by a particular author by examining other writings by that author [3, 10]. Whilst the roots of author identification can be found within a paper- based human-oriented approach, more recently its use within an IT context, where the IT system is performing an automated verification or identification of the user makes this firmly a biometric technique. As previously highlighted, the approach, when automated is analogous to linguistic profiling. 


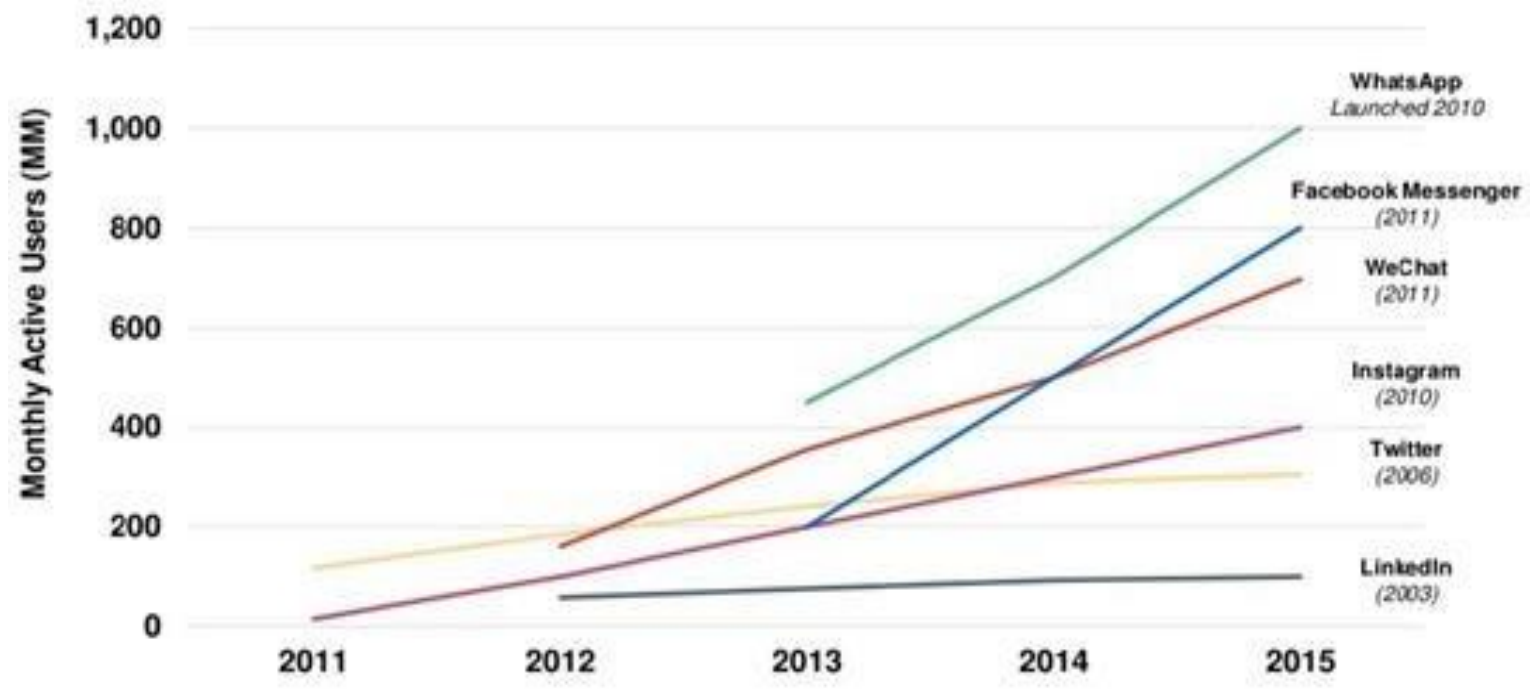

-Linkedin - Twitter -Instagram -WhatsApp - WeChat - Facebook Messenger

Figure 1. Monthly active users on selected social networks

Recently, with the rapid growth of computing, the nature and need for author identification has expanded from written documents into electronic files. Alongside the introduction of computing, came the widespread use of the term stylometry. Stylometry is the study of all features of a document. According to Zheng et al [3] there are three main characteristics of stylometry: Analysis tasks, writingstyle features used, and the techniques investigate these features. Stylometry in the field of author identification is an old research area that go back to pre- computer times. Stylometry is the study of linguistic style including length, word choices, word count, syntactic structure and other related attributes [12].

Biometric systems have been utilized in many security systems to identify and verify the authenticity user from an impostor [13]. While the behavioural biometric methods may be less accurate and unique as they need longer time to extract features of users and can be used as identification techniques to determine the authenticity of the user [14]. Author identification or linguistic profiling is a form of a biometric system that used to identify, and discriminate users based on writing styles. Writing styles is unique and behavior expresses the person's way of writing and plays a large role in the process of demonstrating the user and specifying the language of the person's profile. Many types of linguistic features can be profiled, such as lexical patterns, syntax, structure, content specific, characteristic content. It built on that assumption people have a characteristic pattern of language usage, as a sort of "authorial fingerprint". Exploring the author identification and deriving from the length of the text, the features of the selected vocabulary, the sentences used the structure of messages that was created in different type of message platforms show a main part in providing the reference template used to complete the author identity identification process as Figure 2 show.

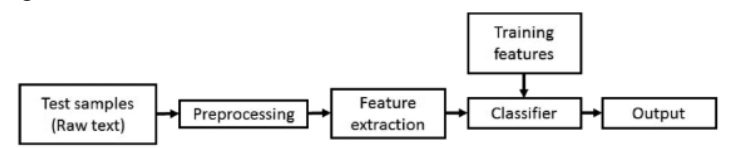

Figure 2. A typical authorship attribution system [15].

A large volume of literature is available on author identification for long documents and little literature exists on using author identification for short texts and focused upon different messaging systems. Most of the techniques used in this area have focused only on identifying users' stylometry in individual messaging systems [12, 16-21]. Some researchers in this field have focused only on using the relationship with the same user's stylometry linked to different messaging systems through a technique known as "linkability" [22]; for example, linking the user's stylometry based on a user profile. Meanwhile, other research has mainly focused on using techniques such as statistical analysis, mining users, clustering and classification.

In addition to the core research questions, the following sub-questions have been identified:

- What is the capability of stylometric features in text limited messages?

- How reliable can identification of short text messages be identified?

- What the nature of the underlying methodology utilized?

- Whether the approach allowed user's profiles can be transferable between systems. 
Figure 3 shows the number of publications returned for each database after several enumeration rounds using this query, and also provide the final number of selected studies.

\begin{tabular}{lcc}
\hline Database & Number of References & Selected References \\
\hline IEEE Xplore & 95 & 8 \\
ScienceDirect & 90 & 8 \\
ACM Digital Library & 130 & 10 \\
SpringerLink & 225 & 7 \\
Google Scholar & 145 & 12 \\
\hline Total & 517 & 45
\end{tabular}

Figure 3. Number of returned references

In addition to the selection criteria, Figure 4 illustrates that the primary publications were classified based on the quality of each study. Published papers and outputs are available in appendix section.

\begin{tabular}{|l|l|}
\hline Genre & Number of Primary Studies \\
\hline Peer-reviewed journal's papers & 24 \\
\hline Peer-refereed book chapters & 6 \\
\hline Peer-reviewed conference papers & 15 \\
\hline Total & 45 \\
\hline
\end{tabular}

Figure 4. Publication's genre and the number of primary studies

On a serious note, the techniques related to author identification have been ignored, such as mouse and keystroke dynamics, since these techniques form an integral part of specific complementary systems, as well as lacking the stylometric features that can be found when linked to other components that work in combination for another system. Similarly, a number of studies that have focused on mining and clustering users have been ignored, except for in some studies linked to stylomerty. These have not been ignored in order to show how authorship analysis operates with it or with unknown users. In addition, due to the lack of features associated between users and messaging systems, the clustering and mining techniques basically depend on categorizing users. Likewise, a number of studies has been ignored since they use the same technique for the same platform of messaging systems, resulting in very similar findings. The outcome of the results of the research has reduced the number of papers from approximately 517 to 45 papers, and these have been categorized according to the most relevant studies.

\section{Literature Review of Author Identification}

According to Zheng [3], the CUSUM statistics tool is used to produce the cumulative sum of the deviation of the measured variable to compare amongst users and it is also employed as a forensic tool to help experts confirming authorship analysis. Based upon most experimental findings and results, it was concluded by most scholars that machine learning methods were more accurate than statistical approaches [11]. According to Nirkhi et al [11] the performance of authorship analysis can be largely influenced by stylometric feature selections; which is used in writing style in order of discovering and finding out the most effective discriminators.

According to Brocardo et al [23] authorship analysis can be seen from three different perspectives. Firstly, author identification finding the author of the document or post in question, given the samples of the writing of a number of authors, the main goal is to conclude which author wrote the document or post in question, and the reliability for the author would be one of those whose samples were given [3]. Secondly, authorship verification involves checking if a target document was written or not by a specific person by investigating other pieces of writings from that person, it gives a binary answer of "Yes" or "No" to the question [23] Li, 2014). Thirdly, concluding the characteristics of an author and deciding the author behaviour (age, gender, race, culture, education, background. etc.). Figure 5 shows the main fields and its tasks in a classification for authorship analysis. As reported by Kebede et al. [24], all the sub-fields of authorship analysis are powerful enough to distinguish a single author from multiple authors by examining the stylometric features.

\begin{tabular}{|l|l|}
\hline \multicolumn{1}{|c|}{ Category } & Description \\
\hline $\begin{array}{l}\text { Authorship } \\
\text { identification }\end{array}$ & $\begin{array}{l}\text { Deciding the most likely author of an anonymous } \\
\text { document by comparing }\end{array}$ \\
\hline $\begin{array}{l}\text { Authorship } \\
\text { verification }\end{array}$ & $\begin{array}{l}\text { Checking whether a target document was written } \\
\text { or not by a specific author. }\end{array}$ \\
\hline $\begin{array}{l}\text { Authorship } \\
\text { characterization } \\
\text { or profiling }\end{array}$ & $\begin{array}{l}\text { Concluding the characteristics of an author and } \\
\text { deciding the author behaviour (age, gender, race, } \\
\text { culture, education, background etc.). }\end{array}$ \\
\hline
\end{tabular}

Figure 5. Classification and taxonomy for authorship analysis [23]

\subsection{Stylometric Features}

The majority of previous studies to date have actually focused only on stylometric features in order to achieve recognition of the authors. In fact, knowing what the best set of features to be used in author identification can be difficult. However, the majority of researchers would combine two or more types of stylometric features [4]. Abbasi and Chen [4] emphasis that there is an urgent need to use larger feature sets that consist of various groups of features for example, punctuation with word-length distributions and combine lexical with syntactic, syntactic and other features. According to $[3,4$, and 12] the use of feature sets containing lexical, syntactic, structural and context-specific features are 
more effective and operational for online identification. The stylometric features are widely used as some previous studies [24] have indicated that many courts of law use it to permit it as evidence in some countries such as Great Britain, United States of America and Australia.

Abbasi et al. [4] suggest stylometric analysis techniques can be classified into two main groups: supervised and unsupervised methods. Supervised techniques refer to the methods involving authorclass labels for categorization. It can be used in investigation fields such as forensics in order to identify criminals as investigators typically have a limited number of identified suspects and thus can identify and capture verified samples from which to compare the suspect message [25]. Whereas unsupervised techniques can be used where this is a lack of any prior knowledge of author classes [26]. Common supervised techniques applied in authorship analysis are support vector machines (SVM) [27], neural networks [3], decision trees [12], linear discriminate analysis [28]. While unsupervised stylometric categorization techniques consist of principal component analysis (PCA) and cluster analysis.

There is another name and commonly known use to identify users that is referred to 'writeprints' [4]. This technique represents an author's writing style, which is frequently consistent across his or her writings, and further become the basis of authorship analysis. These features are gathered from previous works and contain lexical, syntactic, structural, context-specific, and idiosyncratic features [29]. Another development in the field of stylometry is a technique called Doppelganger Finder [30]. This method was specifically designed to link users having multiple accounts within the same forum and has a rather complex framework in terms of how it is implemented [29]. Most of the methods used in previous studies to identify the author of the text are used to mix different features of stylometry in the recognition of authors through their text messages, as well as most of previous researchers emphasized the importance of combining the features of stylometry in order to achieve high accuracy. The following sections will review all the studies and methods used in most previous studies in the use of stylometric features for both long and short text messages.

\subsection{Stylometric Features in Long Text}

As it previously highlighted, many early studies would combine two or more types of stylometric features such as lexical feature (for example, word or character occurrence) and syntactic (such as function words). More recently, renewed focus has been given to identifying different features [32]. For instance, Baayen et al. [28] used 50 common function words and 8 punctuation symbols and tested this using 72 articles written by 8 authors with 908 words per article. The study was conducted in Dutch. The classifier used was entropy-weighted linear discriminant analysis (ELDA). Their method was to measure the degree to which a non-professional author with a similar background can be distinguished on authorial structure in texts and their results showed this to be true with an accuracy of $88.1 \%$.

With the same objective of using a combination of stylometric features, a study by [3] focused upon the identification of online text messages. They demonstrated a method which can be used for multiple-languages. They tested their method on both English and Chinese newsgroup messages. For English language, they collected an average of 48 messages from each of the 20 authors, with an average message length of 169 words. The same authors also test their experience on the Chinese language, with an average of 37 messages per author. For each of these messages has an average of 807 words; The average message length in the Chinese language is bigger than the average message length in English and this is due to the fact that the Chinese is a typical Oriental language which has no word boundaries [3].

A combination of 270 features as follows (87 lexical, 158 syntactic (with 150 function words), 14 structural, and 11 content-specific) was employed. The experiments involved the use of three natural language classifiers: SVM, C4.5 decision tree, back propagation neural network, and $\mathrm{C} 4.5$ decision tree. The overall set of 270 features produced the best performance in term of accuracy over any subset. SVM displayed the best result of $90-97 \%$ accuracy rate for the English data set and $72 \%-88 \%$ for the Chinese data set. In both languages, SVM outperformed both the decision tree and neural network. Since, SVM classifier has the ability to handle large-scale classification in long texts. Structural features and content-specific features demonstrated better in terms of discriminating capabilities for authorship identification on online messages since they show how the author build the content of a message structurally, and contentspecific indicate the level of depth of the author's cultural or a particular domain. For instance, the word "software" has been always used by computer students.

In the same context, Iqbal et al [32] studied 292 stylometric features comprising of lexical, syntactic, structural and topic specific with 158 users and 200 emails each. Their study focuses on to confirm that if a given suspect is the true author of a doubtful textual document or not. They try to use two e-mail datasets; one taken from a large population and the other one taken from potential suspect; each e-mail has been converted into a vector of stylometric features. They tried to use two different methods. 
The first method implements verification using classification; three different classifiers are investigated, Adaboost.M1, Bayesian Network, and lastly Discriminative Multinomial Naïve Bayes (DMNB).

The second method implements verification by regression; three different classifiers have been studied including linear regression, SVM with Sequential Minimum Optimization (SMO), and SVM with RBF kernel. Where the decision that used to verify the author is based on the threshold defined for their suggestion, and the threshold was calculated based on varying the relative number of false positives and false negatives based upon the nature of the supposed application for the system. They stated that authorship analysis outputs on Emails in less than 500 words would not be significant in terms of increasing more performance. Their limitation is that the style variation of the same suspect when he writes may affect his representative model, and they achieved accuracy rate of $80.6 \%$ via a Bayesian Network classifier. However, combining the features of the lexical with the syntactic and with the structure seems to give the highest accuracy in Email because the email is often formal and contains information addressed to the receiver of the message. Thus, the features of the structure, the diversity of words in the text indicated to that the features of the lexical, and the features of the syntactic are all active because most of the emails contains information or explanation such function words ("in", "or", "at", etc.) or punctuation.

Monaco et al. [34] examined 30 book authors with 228 lexical and syntactic stylometric features. Each author had 10 books, and each book had about 10,000 words. The stylometry system uses the stylometric features as follows: 49 character-based, 13 word-based, and 166 syntax-based features. In addition, the features have been selected to show reasonable variation over a population of authors. For example, some authors use a large vocabulary and others a small one. The features have been normalized for instance, the number of different vocabulary words, and the number of words. The 300 text samples have been cut into files of eleven different sizes $(250,500,750,1000,1500,2000$, $2500,3000,4000,5000$ and 10000 words) to get system performance as a function of text length. They used the k-Nearest Neighbour as classifier; and achieved a $91.5 \%$ rate of accuracy in an authorship authentication. However, this shows that the selection of lexical features and syntactic features and their integration with each other gives a high percentage in achieving accurate verification in long texts and especially in documents such as books. Although the differences in number of users and the number of books may have an impact on increasing the accuracy, the classifiers also play a major role in improving accuracy.
In the same context using books with a different classifier, Koppel et al. [35] achieved $95.7 \%$ when used 250 most commonly occurring words that are often named n-gram to authenticate the author among 10 authors using 21 books with texts of varying length for each author and SVM was used as a classification method. This indicates to that the use of n-gram for determining the most used words has also an effective effect in verifying authors of books and long documents, since, it displays the number of words or characteristic used in the text. Although, both studies [34] and [35] used the lexical features to verify the writing style of the users for books and long document, the study conducted by [35] showed a significant increase of accuracy because they used a SVM classifier rather than a KNN classifier, which indicates to that SVM outperform on KNN classifier especially in long text.

Stamatatos [19] used common n-gram features for author identification when limited samples are existed for testing; they investigated the class imbalance problem and conducted an experiment for compensation of imbalanced data sets. The data of 50 authors has been collected from the Reuters Corpus Volume I (RCV1), each author created 100 messages which ranged from $288 \mathrm{~KB}$ to $812 \mathrm{~KB}$ (1KB is roughly 500 words), and using an altered Common $\mathrm{N}-\mathrm{Gram}$ (CNG) method and they achieved an accuracy rate of approximately $70 \%$ by using SVM as a classifier.

More studies on author attribution on imbalanced data proposed that uses many short text samples for the minority classes and less and longer text samples for the majority classes [36]. Therefore, it is necessary to take into account the size of the samples used for testing to evaluate the accuracy when performing the identification process and most studies in this area proved that there is no mechanism and solid to provide the appropriate size of the samples for the testing to be applied in biometrics during the identification process.

Koppel et al. [37] tried to identify authors by using a combination of function words, bi- grams, and 99 idiosyncratic features such as sentence fragments, wrong vowel and mismatched tense. Their proposed was to use syntactic information based on syntactic error and evaluates the effectiveness of such features, both in and of themselves and in combination with other types of features. They performed the study on 480 emails among 11 authors during a period of nearly a year, and each email involving of about 200 words. Three classes of features have been used containing lexical (i.e. function words: "and", "the", "that"), Part-ofSpeech (POS) Tags (i.e. verb, noun) and idiosyncratic usage (i.e. syntactic, formatting and spelling usage).

The appearance of function words can be used as a marker of writing style and could be an indicator of 
authorship. The POS tagger has been employed to the corpus to label each word with one of 59 POS tags and after that, used the frequencies of all POS bi-grams that appeared at least three times in the corpus as POS feature set. The study showed the use of idiosyncratic features significantly improved the accuracy rate from $61.7 \%$ to $71.8 \%$, using decision tree as the classification method. Compared to the study of [32] as mentioned above, and since both used the email as a platform. It has been found that, although the number of users in the study of [32] more than the number of users studied by [20], the use of lexical and syntactic features in the verification/identification of the email is more effective than the use of the idiosyncratic features, because it is possible that the idiosyncratic features has changed during the writing of the email, for example, (English - British) to (English - American). Having said that, the idiosyncratic features of the word "center" instead of "centre", a word "center" frequently used in the United States of America while a word "centre" originally used in the United Kingdom. Second, the use of the bayesian classifier seems better than using decision tree specifically in the email platform since decision trees suffer from fragmentation in such cases-particularly if little data was available [33].

There are a few researchers examine effectiveness of stylometry for authorship authentication and identification with text in a range of 75 to a couple of hundreds of words. For instance, Orebaugh [38] developed an instant message intrusion detection system framework in order to test instant message conversation logs of 4 users based on 69 stylometric features and focuses basically on examining character frequency as a stylometric feature and additional stylometric features were used include: sentence structures, predefined specific characters, emoticons, abbreviations analysis. The study tried to analysis of 2500 characters which approximately 500 words, assuming that $(1$ word $=5$ characters). The naive Bayes classifier has been used and achieved an accuracy rate approximately $68 \%$. The results show that uppercase characters, special characters and numbers are distinguishing, and it can be used as a form of intrusion detection system. According to Ali et al [39] identifying and showing these features are the main challenge for authorship identification. Since it has emoticons, special characters and uppercase or lowercase.

In the same context of using limited words, in gender identification which is a branch to the authorship problem, Corney et al [40] investigated 4 users; each user has 253 e-mails and ranged from 50-200 words per e-mail. They used function words, structural, stylistic, gender attributes features and SVM has been used as a classification engine, they achieved accuracy approximately $70.2 \%$. Their approach distinguishes between male and female authors. The main finding of Corney's outputs, function words provide the most important aspect for discriminating gender. Cheng et al [41] observed that gender identification problem can be treated as a binary classification problem such as class (1) for male and class (0) for female.

Previous studies on long texts especially books, online articles, electronic forums and email, the most commonly stylometric features used for authorship studies were lexical (such as word or character frequency) and syntactic (such as function words or punctuation). One of the most significant findings in previous studies for long documents is that the authorship attribution problem has been significantly influenced by using combination of stylometric feature which combine two or more types of stylometric features. Among various combination features were used, combination of lexical with syntactic can be the best approach to identify authors in long documents and more applicable. Because of the variety of words used in long messages and explain the message in the words used use ("but", "although", "at", etc.).

Significant finding in previous studies for long documents is that authorship attribution has been mainly influenced by the machine-learning paradigm. Among different classification techniques, SVM and Bayesian classifier was regularly used. SVM classifier seems better than Bayesian classifier and decision tree, and Bayesian seems outperform on decision tree. In general, the performance in different type of long documents achieved an accuracy rate of $70 \%$ to more than $90 \%$ for $50-200$ words. Table 1 categorized most studies used in previous studies including techniques, type of features, classification and accuracy.

\subsection{Stylometric Features on Short Text}

This section focusses upon studies that have sought to specifically use short messages. Short text messages are defined in most previous studies based on approximately 75 words or less (look at the comparison features in the Table 2 in discussion section). Instant messages, SMS messages, social network messages are typical shorter messages unlike other online messages or posting like blog posts or online articles. As it can be clearly shown above in stylometry of long texts, the traditional stylometric features, particularly lexical, syntactic features or combinations of them are more applicable. However, with microblogs or social network messaging systems, users could just post a message as a quick update of their status or activity they are involved in. Twitter is one of the social networks that use a restriction on the amount of text which restricts its user by using only 140 character maximum. 
Table 1 . The summary of literature review of stylometry searches with long text besides its accuracy

\begin{tabular}{|c|c|c|c|c|c|}
\hline Author & $\begin{array}{c}\text { No. of } \\
\text { Suspects }\end{array}$ & Feature Types & Classification type & Accuracy & Goals of study \\
\hline Zheng et al. 2006 & 20 & $\begin{array}{l}\text { Lexical, structural, } \\
\text { Syntactic, and } \\
\text { content specific }\end{array}$ & $\begin{array}{l}\text { SVM decision tree, } \\
\text { and NN }\end{array}$ & $\begin{array}{l}97.69 \% \text { for SVM, } \\
96.66 \% \text { for NN and } 93.36 \% \text { for } \\
\text { C4.5 }\end{array}$ & identification \\
\hline Tan, et al. 2010 & 2 & $\begin{array}{c}13 \text { Syntactic and } 4 \\
\text { lexical }\end{array}$ & Naïve Bayes & $81.98 \%$ & Identification \\
\hline Steyvers, et al.2004 & 85 & $\begin{array}{c}\text { Author- topics and } \\
\text { topic- } \\
\text { word models }\end{array}$ & SVM & $72 \%$, & $\begin{array}{c}\text { Topic } \\
\text { discovery }\end{array}$ \\
\hline Stamatatos 2007 & 50 & Common n-gram & SVM & $70 \%$ & Identification \\
\hline Pavele, et al.2009 & 20 & $\begin{array}{l}\text { Conjunctions and } \\
\text { adverbs }\end{array}$ & $\begin{array}{c}\text { Prediction by partial } \\
\text { matching (PPM), and } \\
\text { SVM }\end{array}$ & $\begin{array}{c}\text { 83-86\% for PPM } \\
82.9-84 \% \text { for SVM }\end{array}$ & Identification \\
\hline Monaco, et al.2013 & 30 & $\begin{array}{c}\text { Lexical and } \\
\text { syntactic }\end{array}$ & K-NN & $91.5 \%$, EER 8.5 & Authentication \\
\hline Koppel, et al.2004 & 10 & $\begin{array}{c}\text { Common words or } \\
\text { partial } \\
\text { word (n-gram) }\end{array}$ & SVM & $95.7 \%$ & Authentication \\
\hline Iqbal, et al. 2010a & 158 & $\begin{array}{c}\text { Lexical, syntactic, } \\
\text { and structural }\end{array}$ & Bayesian network & $80.6 \%$, EER 19.4 & Authentication \\
\hline Corney, et al. 2002 & 4 & $\begin{array}{c}\text { structures, stylistic } \\
\text { function words and } \\
\text { gender- attributes } \\
\end{array}$ & SVM & $70.2 \%$ & Gender discovery \\
\hline Baayen, et al. 2002 & 8 & $\begin{array}{c}\text { 50 Function words, } \\
8 \\
\text { punctuation } \\
\end{array}$ & $\begin{array}{l}\text { Entropy- weighted } \\
\text { linear }\end{array}$ & $88.1 \%$ & Identification \\
\hline Orebaugh 2006 & 4 & $\begin{array}{l}\text { Sentence structure, } \\
\text { emoticon, and } \\
\text { abbreviation. etc. }\end{array}$ & Naïve Bayes & $99.29 \%$ & Identification \\
\hline Howedi,et al. 2014 & 10 & $\begin{array}{c}\text { Lexical, structural, } \\
\text { Syntactic, and } \\
\text { content specific } \\
\text { character N-gram } \\
\end{array}$ & $\begin{array}{c}\text { Naïve Bayes and } \\
\text { SVM }\end{array}$ & $96 \%$ & identification \\
\hline Ragel et al.(2013) & 70 & unigrams & \begin{tabular}{|l|} 
cosine similarity and \\
the Euclidean \\
distance
\end{tabular} & $25 \%$ & Identification \\
\hline
\end{tabular}

Therefore, certain stylometric features, as structural features, may not be applicable and effective to work because, users do not have so much control over the content of the post and will indeed potentially need to modify their behaviour in order to conform to the restrictions placed upon the messaging platform.

Layton, et al [16] tested 50 Twitter users with each user having 120 Tweets. They used a 3-gram approach and the Source Code Authorship Profile (SCAP). The study obtained an accuracy of $70 \%$. The users of Twitter can use "\#” followed by a tag name to link messages with specific topics. Also, the users of Twitter heavily use "@” followed by a specific user's name to direct the message to a destination. All these structural contents were counted toward the 140-character limitation; however, all these structural contents were removed by the researchers before applying the SCAP algorithm to allow SCAP to focus on the actual content that the user made and they point out that 120 tweets per user is an important threshold. Furthermore, the SCAP method extends [42] on classification [43]. In Keselj's study [42], an author profile is described as "a set of length $L$ of the most frequent n-grams with their normalized frequencies." and an n-gram is an numbers of characters in contiguous sequence. The profile of an author can be indicated as $\{(\mathrm{x} 1 ; \mathrm{f} 1),(\mathrm{x} 2 ; \mathrm{f} 2) \ldots(\mathrm{xL} ; \mathrm{fL})\}$ For $\mathrm{i}=1 \ldots \mathrm{L}$, where $\mathrm{xi}$ indicates to an $\mathrm{n}$-gram and fi indicates to the normalized frequency of xi. For SCAP, the frequency fi of the n-gram xi was not normalized. A profile of an author is defined as the $\mathrm{L}$ numbers of n-grams which have the highest frequency, which can be as: $\{x 1, x 2 \ldots x L\}$. Throughout the classification process and when an unknown profile was accessible for authorship identification, the author who shared the most ngrams with the unknown profile will be specified as the author of the unknown profile. However, the drawback in their method is that any increasing in messages would not have any positive effect on the accuracy, where their method was questioned in relation to the authorship identification task of not so common messages as the accuracy rate dropped by $27 \%$ when data about the discusser's user information was taken out [44]. 
Table 2. The summary of literature review of stylometric features on short text besides its accuracy

\begin{tabular}{|c|c|c|c|c|c|}
\hline Author & $\begin{array}{c}\text { No. of } \\
\text { Suspects }\end{array}$ & Feature Type & Classification & Accuracy & Goals of study \\
\hline Layton, et al. 2010 & 50 & Character n-grams & $\begin{array}{c}\text { SCAP } \\
\text { Algorithms. }\end{array}$ & $70 \%$ & Identification \\
\hline Green, et al. 2013 & 12 & $\begin{array}{c}\text { Bag-Of- Words and style } \\
\text { markers }\end{array}$ & SVM & $\begin{array}{c}12 \text { users were } \\
\text { tested gain } 40.5 \% \text {, }\end{array}$ & Identification \\
\hline Allison,et al. 2008 & 9 & $\begin{array}{l}\text { Word frequency, 2-grams, 3- } \\
\text { grams and stem words }\end{array}$ & $\begin{array}{c}\text { Multimodal } \\
\text { Hierarchical } \\
\text { SVM }\end{array}$ & $\begin{array}{l}78.46 \% \\
87.05 \% \\
86.74 \% \\
\end{array}$ & Identification \\
\hline Zheng, et al. 2006 & 20 & Lexical Syntactic, structural & SVM and C4.5 & $\begin{array}{c}97.69 \% \text { and } \\
93.36 \%\end{array}$ & Identification \\
\hline
\end{tabular}

One of the most significant technique to identify the author in Twitter is to deal with n-gram to create the reference template which containing a contiguous sequence of $\mathrm{n}$ items of a particular sequence of text which has the power to collect and distinct the characters of Twitter as long as the limits of characters in Twitter is 140.

Similarly, Green et al [45] studied authorship identification in Twitter, and collected data from only 12 users, with 120- 900 tweets per user. The feature set used comprised of: Style Markers and Bag-of Words (BOW). The number of style markers utilized was 86 and contains punctuation, long word, part of speech, hyperlinks, and other similar attributes. The BOWs contain all the words that come from the raw data which used as a measure when the words appeared more than 5 times in the whole dataset. SVM were used as a classifier and, Sequential Minimal Optimization (SMO) in WEKA was used as algorithm, and a linear kernel has been used as a parameter. They found out that Style Markers performed better than BOWs for short text with an accuracy ranging from $60 \%$ to $76.75 \%$ for BOWs, and $75.1 \%$ to $92.3 \%$ for Style Markers. The drawback is that when the researchers examined the effect of the number of authors found out that the accuracy decreased from $92.3 \%$ with two more authors were added to become $40.5 \%$ with 12 authors. The reason for the low accuracy rate is that the greater the number of users added the lower the accuracy rate. The increase in the number of users has a significant impact on the parameters process especially with the Style Markers. Because each new user seems to have new patterns in writing style that affects the stability of the overall measurement specified in the balancing parameters. Features of style markers may be suitable to identify authors in small samples of dataset because it is possible to be weak when the number of authors increases [46].

In comparison to the previous study [16], it can be inferred that Layton et al.'s their accuracy dropped to approximately $27 \%$, while Green et al's decreased accuracy was approximately $51 \%$, taking into account that the number of authors at Layton is bigger four times in compared to the number of authors at Green, which indicates that n-gram can play an important role and more effective in Twitter. Because the n-gram has the ability to handle characters and has the ability to distinguish them within text, this leads to user identification.

Ragel et al. [21] focused specifically on authorship detection of SMS to identify authorship using unigrams as features. They stated that the length of the SMS is limited to 140 characters. NUS corpus [47] has been used since it contains more than fifty thousand SMS messages written in English. They used two methods cosine distance as well as Euclidean distance in order to choose a suitable comparison method. 20 authors have been used and more than five hundred SMS each. The first method, a unigram authorship profiling has been used and a distribution of unigrams has been created for each author. Furthermore, the extracted unigram profiles compared against the training set. The author is determined based on similarity comparison between the training and the testing data via the cosine and Euclidean distances. The second method, they tried to determine the effect of the size of the training set. They noticed that when the number of possible authors increases, the decrease in the accuracy is close to linear. They sum up with that ten stacked SMS messages is ideal for the close best accuracy to detect the author of a set of SMS messages using cosine similarity distance metric with unigram method. The drawback is that their results demonstrated a low precision of below $25 \%$, among only 70 SMS users. However, this can reflect the difficulty of obtaining a high precision in authorship identification of SMS users and more appropriate to use another effective technique such as a Part-ofSpeech POS combined with n-gram to decrease the number of features.

Allison et al. [18] focused on author identification of email. They investigated 9 users on short emails with approximately 75 words each and with a range of 174 to 706 emails per user, Enron Email corpus has been used and used 2-grams, 3grams and word frequency measures. SVM was used as the classification engine, which produced around 
$86.74 \%$ accuracy. In comparison with the previous studies mentioned using the e-mail platform for short text, [18] and [40] both utilized SVM as a classifier, Allison achieved $86.74 \%$ while Corney et al. [40] achieved $70.2 \%$, Keep in mind that the message length of Allison is 75 words and Corney's message length is between 50-200 words and the differences is only in the number of users and the number of chosen features in stylometry. This indicates to that there is a weakness to determine the best features to be used in stylometry, the best size of the e-mail message, and the appropriate number of users of email platform. In order to be used in the identification process. This leads to discover and to find out the optimization for features mentioned above, because there is no solid base for reliance on during the investigation or exploration.

Koppel et al. [48] and Sanderson et al. [49] investigated 500 words from books and newspaper journalists, respectively. They used an approach "Author Unmasking", the idea of author unmasking is that the differences between two texts from the same author will be reflected in a relatively small number of features. These features can be extracted by use of an author unmasking curve. In Koppel et al.'s experimental, they selected the 250 most frequent words and the collection of 21 English books published in nineteenth century has been used. These books were written by ten different authors. Each book has been chunked into equal sections of at least 500 words without breaking up paragraphs. A linear-kernel SVM has used for cross-validation; they obtain overall accuracy of $95.7 \%$. In Sanderson et al's experimental, they used 50 newspaper journalists, and the number of authors about 50 authors with a minimum of 10,000 words per journalist, they divided the training set into 500 characters per chunk used. A Linear SVM classifier has been used and the accuracy achieved over $90 \%$. They suggested that there is noteworthy aliasing between different-author and same-author performance curves when considering samples of 5,000 words or less and stated that the author unmasking approach is less useful when dealing with short texts. They concluded that measuring of the "depth of difference" between two example sets is a different type than other measures, such as margin width, that could be based on a single highly differentiating feature and it is not appropriate this measure should be applicable to other applications.

Siham and Halim [50] also maintained the idea that the longer the text, the better the identification accuracy will be. The possibility of short documents analysis is difficult to carry out since this type of document is usually characterized by a poor structure and an informal language much less seen in literary texts [23]. For example, the content of the short message that exists in social networks such as Twitter and Facebook is often written without author's conscious; this is due to the fact that the writing practice provided by the platforms does not allow more contents and may be restricted by certain linguistic restrictions, thus, the text message can be unclear language and unorganized format.

In general, the researchers did not agree on a clear vision and a general framework used when the message is being poorly structure and uninformally language built to determine its validity and its suitability when it is being used in the investigation process. For example, the length of the required message, weakness, and strength of stylometric features as well as acceptable format. Having said that, the number of the research that were conducted on short texts is smaller in comparison to the research on long texts. The majority of the studies on short text are associated to digital copies, for instance, emails or social network posts, ranging from 140- character text for Twitter to emails of 75 words. Lexical and syntactic features were commonly used on short texts; SVM can be a common classification method.

Most of the previous studies showed that the effect of lexical and syntactic is an important factor in short text messages for the following reasons: Firstly, short text messages attempts to be a summary and specific to be understood by the other party and try to be inclusive of a few words so the author of the letter takes into account the rich words intended. Secondly, Short text messages contains rules for the sentence to be adopted by adding words such as (but, "and","on","therefore", etc.) As well as punctuation. This plays an important role in the process of syntax the text message because most of the texts contain comprehensive and accurate explanation. Thus, for example, a Twitter message containing 140 characters and the purpose of delivering the text message to the other party in a conceptual and concise. Technically, the most characteristic feature to deal with short messages is n-gram because it plays a large role in determining the characters of letters-words in sentences. As we have seen, most previous studies focused on the n-gram and have achieved a high level of accuracy.

The performance of authorship attribution systems on short texts can be affected by several factors. Stamatatos [19] and [3] investigated the effectiveness of parameters in identification for example, how many authors can be expected to be identified as well as the number of messages that can have an influence on recognition performance. Zheng, et al [3] states that in the authorship analysis task the texts should be attributed to a single or more predefined classes based on the authors which means that these texts are grouped into different classes by their authors. 


\section{Discussion}

This part is divided into two sections. First section discusses the mechanism of identifying authors in long text messages as well as short text messages. Section two discusses the mechanism of connecting authors in multiple platforms.

Part I: Discusses the mechanism of identifying authors in long text messages as well as short text messages.

\subsection{Identification of authors for long text messages}

Regarding identification of authors on long texts especially books, online articles, electronic forums and email, the highest number of users was used, goes for the study [32] used about 158 users compared to all previous studies using the email as a platform and 200 e-mail for each users, the sample size was less than 500 words, features type were lexical, syntactic, and structural, 292 number of features were used by using the Bayesian network and achieved accuracy rate of $80.6 \% \%$, their limitation is that the style variation of the same suspect when he writes may affect his representative model. While the lowest number in the number of users were 2 users for the study [52] using 167 blog posts per user, 170-357.5 words sample size, the features types were 13 syntactic and 4 lexical, 21, No.of features were 21 using Naïve Bayes as a classifier, Their limitation is that each author exhibits differences in the length of their entries in the database in terms of word count and achieved accuracy of $81.98 \%$.

Although the difference between the two studies in the number of users and platforms and both of them used lexical and syntactic, but [32] used the feature of structural, and this seems to increase the rate of accuracy and plays an important role in the email platform even if the number of users are large and this features can be the best features because it reveals the manner users style while using email as a platform, while in the Tan's study, they did not use this feature although the number of users is less. The reason for the absence of this features in the Tan's study is that most users of blog post do not consider these features as long as users write randomly without any conscious and do not write official text messages such as email. The best performance can be for the study [32] because they deal with a large number of users and even the accuracy of the study [52] was slightly increased by almost $1.38 \%$.

From another side at using the email, it can be noted that (Corney et al.) [40] used the email as a platform identification, the number of users 4 , samples each user 253 email, the sample size 50-200 words, the features type were structures, stylistic, function words and gender- attributes, No of features 22 , the classifier used SVM and achieved $70.2 \%$. Their limitation they mentioned that there is a need for larger set of gender attributes to be used in order to increase the performance results. Although the study [40] and the study [32] both use email as a platform, and despite their differences between them in number of users, features type and classification. In fact, function word feature in the study (Corney et al.) [40] played a major role in increasing accuracy in Corney's study, and keep in your consideration Iqbal's study uses structure feature.

Koppel et al. [20] and Monaco et al. [34] both used the book, the number of users in Koppel, et al.'s study was 10 users, samples each suspect 21 books, the sample size about 500 words per chunk, the type of feature used is common word (n-gram), the number of features 250, the SVM as classification type was used their limitation is that unmasking approach might find more general application, and achieved the accuracy of $95.7 \%$. While in the study [34] 10 books were used, No of users was 30, the sample size was 10,000 words, the features used were lexical and syntactic, the number of features was 228, and the classifier was K-NN, their limitation is that the database is relatively small, and achieved accuracy $91.5 \%$.

In general, the most commonly stylometric features used for author identification were lexical (such as word or character frequency) and syntactic (such as function words or punctuation). One of the most significant findings in previous studies for long documents is that the authorship attribution has been significantly influenced by using combination of stylometric features which combine two or more types of stylometric features, since combination of stylometric features, could help to improve the accuracy of identification, and contains lots of features. The longer the text is, the easier it is to compute stylometric features, which become more reliable as more text is considered. Among various combination features were examined, it seems that the combination of lexical with syntactic have achieved high accuracy in identifying authors in long documents and can be more applicable.

\subsection{Identification of authors in the short text messages}

The second part of this review has focused on identification of authors on short texts. The number of the research are limited. The majority of the studies on short texts have been applied to emails or social network posts, ranging from 140-character text for Twitter to emails of 75 words. Except for the study [3] ranging 84-346 words, which is a slightly higher than authors' approach since Zheng's samples for each suspect was less between 30-92 Email samples. This gives the indicator that increasing the 
number of suspects' samples for short text message is also an important factor for message size, because the greater the number of author's samples the greater the recognition rate is achieved and vice versa.

From another point of view, Layton et al. [16] and Green, et al. [45], both have used Twitter as a test platform. The aim of their study is identifying users on Twitter, the type of features used in the study [16] was n-gram, No.of users 50, samples for each users 120 tweets, sample size 140 chars max, features type n-grams, no of features was not specified, SCAP algorithms was used as a classifier, their limitation is that the accuracy dropped by $27 \%$ when data about the discusser's user information was taken out. Where the study [45] their aims comparing frequency and style-based features for Twitter author identification, they used the feature of Bag-OfWords and style markers, No of users was 12 , samples for each users 120-900 tweets, samples size 140 chars max, No.of features were hundreds features of bag- of-words and 86 style markers, classifier type was SVM, the achieved $40.5 \%$. This gives the indication that the type of selected features play significant role in increasing and decreasing the accuracy of identification. Since, the features of style markers may be suitable to identify authors in small set of samples and it is possible to be weak when the number of authors increases,

On the other hand, in the study of [18] aim authorship attribution of e- mail, they used the features word frequency, 2-grams, 3-grams and stem words, samples for each user is 9 and classifier are multimodal, hierarchical and SVM. The two classifiers multimodal and hierarchical are probabilistic, that they derive an explicit estimate for the probability which a new document be appropriate to each of the likely classes, No. of Features is not specified, sample size was 75 , their limitation is that complex linguistic features do not allow for successful discrimination, their accuracy were $78.46 \%$ for multimodal, $87.05 \%$ for hierarchical and $86.74 \%$ for SVM. Unigram feature seems to be outperform to bigrams, and to trigrams as long as some certain stylistic texts are doubtless and since they captured by the longer $\mathrm{n}$-grams and can contain more characteristics and this may contribute to the process of getting closer to the identification.

In general, lexical and syntactic features were also commonly used on short texts. Indeed, the unique structural characteristics of messaging systems can also facilitate authorship identification by using these structure and can be important evidences leads to identification and discriminators, for instance, words of beginning of the sentences, greetings, signatures, quotes, links, and could have more important information details lead to understand more about the author. But in case of use in Twitter might be ineffective since Twitter users write informally and randomly and restricted only by the number of specified words.

\section{Part II: Discusses the mechanism of connecting authors in multiple platforms.}

This technique is almost new and there are few studies, and it has been reviewed and discussed extensively previously in the literature studies section. The first work on user linking was conducted by Zafarani et al. [53] who tried to connect users across multiple websites, two methods were suggested, URL of a user profile page which contained the corresponding users name and the natural user's profile which contained another community's username. Liu et al. [54] try to build behaviour similarity model and structure information model, and use multi-objective optimization with missing information to identify linkage across social networks. Afroz et al. [30] try to link users that have multiple accounts within the same forum or blogbased site, linking was based on artificially created accounts of the same user. Almishari et al. [22] try to link Twitter accounts based on very lexical features. However, there is a weakness in all these studies for these reasons:

1. The process of linking users depends mainly on the size of the amount of content of the text message, because often the goal of social networking sites depends heavily on messaging and text messages, even with sites that offer video and image services, for example, the comments in the YouTube video is text, Snapchat provides text messaging service, most social networking sites, email and SMS all provide text, thus optimization text with the number of words with each platform and with multiple platforms were not available in all above studies.

2. The nature of the stylometric features for all platforms need be adapted to each other and optimized with stylometric features types with all platforms. For example, the features of stylometry associated with Facebook, Twitter, or the extent of correlation features with each other, alternatively, this also makes it adaptable to the volume of text messages received in order to carry out the identification process and this is also not available in the above studies. According to Goga et al [55]; Jain et al [56] their work used attributes without analyzing their properties and their limits to match profiles in practice and thus they use attributes with low availability which can only match a small portion of profiles across a small number of social networks and is likely to give many false matches in practice.

\section{Conclusion}

Several methods and systems have been proposed for solving the problem of author identification of 
short texts, it is still not clear what the volume of message needs to be for reliable and confident verification/identification. The drawback of the previous studies is that they have only been focused upon a single platform, yielding incomplete data and insufficient reliability of recognition when dealing with multiple messaging systems, because most platforms differ from each other, whether technically or linguistically and there are several differences in most multiple platforms on different aspects, for example, in modality, Twitter is public platform in nature, while SMS mostly is used for exchanging private text messages. The most significant aspect, linguistically, the post/tweets text message is not necessarily restricted by caution or fear of people such as SMS messages since they are public and the users can easily post and tweet and can hide themselves without any cost, while the SMS has to have or buy a SIM to start anonymity and they takes into account their anonymity and caution because SMS text message has to be addressed to the user and take the linguistic reserve and consideration about it, so he/she cannot be denied in most cases because he/she is the one who places the address of the message to a practically user or specific group of people.

Finally, the drawback of most previous studies is that they have used multi-objective in order to optimize with only with missing information, yielding unsatisfactory data and inaccurate data to achieve the flexibility and the reliability to deal with these platforms. Therefore, there is a lack of understanding about to what extent these stylometric features are actually portable between messaging system platforms. In addition, lexical and syntactic using n-gram is being the most likely candidates to apply in multiple platforms. Indeed, analysis of lexical and syntactic features across different platforms is important in order that the resulting profile is useful across multiple platforms.

\section{References}

[1] Marques, O. (2016). Social Networks. In Innovative Technologies in Everyday Life (pp. 31- 44). Springer International Publishing.

[2] The Guardian (2014, January 12). OMG! Number of UK text messages falls for first time. Retrieved 2017, from https://www.theguardian.com/technology/2014/jan/13/num ber-text-messages-sent-britain- falls-first-time, (Access Date: 19 February, 2019).

[3] Zheng, R., Li, J., Chen, H., and Huang, Z. (2006).A framework for authorship identification of online messages: Writing-style features and classification techniques. Journal of the American Society for Information Science and Technology, 57(3), 378-393.

[4] Abbasi, A., and Chen, H. (2008).Writeprints: A stylometric approach to identity-level identification and similarity detection in cyberspace. ACM Transactions on Information Systems (TOIS), 26(2), 7.

[5] Parameswaran, M., and Whinston, A. B. (2007). Social computing: An overview. Communications of the Association for Information Systems, 19(1), 37.

[6] Stringhini, G., Kruegel, C., and Vigna, G. (2010, December). Detecting spammers on social networks. In Proceedings of the 26th annual computer security applications conference (pp. 1-9). ACM.

[7] Page, R., Barton, D., Unger, J., and Zappavigna, M. (2014). Researching Language and Social Media. New York: Routledge.

[8] Weir, G. R., Toolan, F., and Smeed, D. (2011). The threats of social networking: Old wine in new bottles. Information security technical report, 16(2), 38-43.

[9] Maheswaran, M., Ali, B., Ozguven, H., and Lord, J. (2010). Online identities and social networking. In Handbook of Social Network Technologies and Applications (pp. 241-267). Springer US.

[10] Nirkhi, S. M., Dharaskar, R. V., and Thakare, V. M. (2015). Authorship Identification using Generalized Features and Analysis of Computational Method. Transactions on Machine Learning and Artificial Intelligence, 3(2), 41.

[11] Nirkhi, S. M., Dharaskar, R. V., and Thakre, V. M. (2012, June). Analysis of online messages for identity tracing in cybercrime investigation. In Cyber Security, Cyber Warfare and Digital Forensic (CyberSec), 2012 International Conference on (pp. 300-305). IEEE.

[12] Abbasi, A., and Chen, H. (2005).Applying authorship analysis to extremist-group web forum messages. Intelligent Systems, IEEE, 20(5), 67-75.

[13] Alotaibi, S., S. Furnell, and N. Clarke, Transparent authentication systems for mobile device security: A review. In the 10th International Conference for Internet $\mathrm{T}$ technology and Secured Transactions (ICITST) (pp.406413). IEEE. 2015

[14] Alotaibi, S. and Alruban, A. (2017), Systematic Literature Review of Behavioural Profiling for Smartphone Security: Challenges and Open Problems.

[15] Neal, T., Sundararajan, K., Fatima, A., Yan, Y., Xiang, Y., and Woodard, D. (2018). Surveying stylometry techniques and applications. ACM Computing Surveys (CSUR), 50(6), 86.

[16] Layton, R., Watters, P., and Dazeley, R. (2010, July).Authorship attribution for twitter in 140 characters or less. In Cybercrime and Trustworthy Computing Workshop (CTC), 2010 Second (pp. 1-8). IEEE.

[17] Fridman, A., Stolerman, A., Acharya, S., Brennan, P., Juola, P., Greenstadt, R., and Kam, M. (2013). Decision fusion for multimodal active authentication. IT Professional, 15(4), 29-33. 
[18] Allison, B., and Guthrie, L. (2008, May). Authorship Attribution of E-Mail: Comparing Classifiers over a New Corpus for Evaluation. In LREC.

[19] Stamatatos, E. (2007, September). Author identification using imbalanced and limited training texts. IN Database and Expert Systems Applications, 2007.DEXA'07. 18th International Workshop on (pp. 237241). IEEE.

[20] Koppel, M., Schler, J., and Argamon, S. (2013). Authorship Attribution: What's Easy and What's Hard.

[21] Ragel, R., Herath, P., and Senanayake, U. (2013, December). Authorship detection of SMS messages using unigrams. In Industrial and Information Systems (ICIIS), 2013 8th IEEE International Conference on (pp. 387-392). IEEE.

[22] Almishari, M., Kaafar, D., Oguz, E., and Tsudik, G. (2014 a, November). Stylometric Linkability of Tweets. In Proceedings of the 13th Workshop on Privacy in the Electronic Society (pp. 205-208).ACM.

[23] Brocardo, M., Traore, I., Saad, S., and Woungang, I. (2014). Verifying Online User Identity using Stylometric Analysis for Short Messages. Journal of Networks, 9(12), 3347-3355

[24] Juola, Patrick (2006). "Authorship Attribution". Foundations and Trends in Information Retrieval.

[25] Mariappan, P., Padhmavathi, B., and Teja, T. S (2016). Digital Forensic and Machin Learning. In Combating Security Breaches and Criminal Activity in the Digital Sphere (pp. 141-156). IGI Global.

[26] Khanum, M., Mahboob, T., Imtiaz, W., Ghafoor, H. A., and Sehar, R. (2015). A Survey on Unsupervised Machine Learning Algorithms for Automation, Classification and Maintenance. International Journal of Computer Applications, 119(13).

[27] Li, J., Zheng, R., and Chen, H. (2006). From fingerprint to writeprint. Communications of the ACM, 49(4), 76-82.

[28] Baayen, H., van Halteren, H., Neijt, A., and Tweedie, F. (2002, March). An experiment in authorship attribution.In 6th JADT (pp. 29-37).

[29] Overdorf, R., Dutko, T., and Greenstadt, R. (2014). Blogs and Twitter Feeds: A Stylometric Environmental Impact Study.

[30] Afroz, S., Islam, A. C., Stolerman, A., Greenstadt, R., and McCoy, D. (2014, May). Doppelgänger finder: Taking stylometry to the underground. In Security and Privacy (SP), 2014 IEEE Symposium on (pp. 212-226). IEEE.

[31] Narayanan, A., Paskov, H., Gong, N. Z., Bethencourt, J., Stefanov, E., Shin, E. C. R., and Song, D. (2012). On the feasibility of internet-scale author identification. In Security and Privacy (SP), 2012 IEEE Symposium on (pp. 300-314). IEEE.
[32] Iqbal, F., Khan, L. A., Fung, B., and Debbabi, M. (2010a). E-mail authorship verification for forensic investigation. In Proceedings of the 2010 ACM Symposium on Applied computing (pp. 1591-1598).ACM.

[33] Caragea, D. (2004). Learning classifiers from distributed, semantically heterogeneous, autonomous data sources (Doctoral dissertation, Iowa State University).

[34] Monaco, J. V., Stewart, J. C., Cha, S. H., and Tappert, C. C. (2013, September). Behavioral biometric verification of student identity in online course assessment and authentication of authors in literary works. In Biometrics: Theory, Applications and Systems (BTAS), 2013 IEEE Sixth International Conference on (pp. 1-8). IEEE.

[35] Koppel, M., and Schler, J. (2004, July).Authorship verification as a one-class classification problem. In Proceedings of the twenty-first international conference on Machine learning (p. 62).ACM.

[36] Vorobeva, A. A. (2016, April). Examining the performance of classification algorithms for imbalanced data sets in web author identification. In Open Innovations Association and Seminar on Information Security and Protection of Information Technology (FRUCT-ISPIT), 2016 18th Conference of (pp. 385-390). IEEE.

[37] Koppel, M., and Schler, J. (2003, August). Exploiting stylistic idiosyncrasies for authorship attribution. In Proceedings of IJCAI'03 Workshop on Computational Approaches to Style Analysis and Synthesis (Vol. 69, p. 72).

[38] Orebaugh, A. (2006, October). An Instant Messaging Intrusion Detection System Framework: Using character frequency analysis for authorship identification and validation. In Carnahan Conferences Security Technology, Proceedings 2006 40th Annual IEEE International (pp. 160-172).IEEE.

[39] Ali, N., Hindi, M., and Yampolskiy, R. V. (2011, October). Evaluation of authorship attribution software on a Chat bot corpus. In Information, Communication and Automation Technologies (ICAT), 2011 XXIII International Symposium on (pp. 1-6). IEEE.

[40] Corney, M., De Vel, O., Anderson, A., and Mohay, G. (2002). Gender-preferential text mining of e-mail discourse. In Computer Security Applications Conference, 2002. Proceedings. 18th Annual (pp. 282-289). IEEE.

[41] Cheng, N., Chen, X., Chandramouli, R., and Subbalakshmi, K. P. (2009, March). Gender identification from e-mails. In Computational Intelligence and Data Mining, 2009. CIDM'09. IEEE Symposium on (pp. 154158). IEEE.

[42] Keselj, V., Peng, F., Cercone, N., and Thomas, C. (2003, August). N-gram-based author profiles for authorship attribution. In Proceedings of the conference pacific association for computational linguistics, PACLING (Vol. 3, pp. 255-264).

[43] Frantzeskou, G., Stamatatos, E., Gritzalis, S., Chaski, C. E., and Howald, B. S. (2007). Identifying authorship by 
byte-level n-grams: The source code author profile (scap) method. International Journal of Digital Evidence, 6(1), 118.

[44] Rappoport, R. S. O. T. A., and Koppel, M. (2013). Authorship attribution of micro-messages.

[45] Green, R and Sheppard, J. (2013) "Comparing Frequency- and Style-Based Features for Twitter Author Identification," Proc. Twenty-Sixth International Florida ArtificialIntelligenceResearchSocietyConference, 2013, https://www.aaai.org/ocs/index.php/FLAIRS/FLAIRS13/p aper/viewFile/5917/6043, (Access Date: 19 February, 2019).

[46] MacLeod, N., and Grant, T. (2012). Whose Tweet? Authorship analysis of micro-blogs and other short-form messages.210-224.

[47] Chen, H. (2011). Dark web: Exploring and data mining the dark side of the web (Vol. 30). Springer Science and Business Media.

[48] Koppel, M., Schler, J., and Bonchek-Dokow, E. (2007). Measuring differentiability: Unmasking pseudonymous Authors, Journal of Machine Learning Research, 8, 1261-1276.

[49] Sanderson, C., and Guenter, S. (2006, July). Short text authorship attribution via sequence kernels, Markov chains and author unmasking: An investigation. In Proceedings of the 2006 Conference on Empirical Methods in Natural Language Processing (pp. 482-491). Association for Computational Linguistics.

[50] Siham, O. and Halim, S. (2012). Authorship Attribution of Ancient Texts Written by Ten Arabic Travelers Using a SMO-SVM Classifier, the 2nd International Conference on Communications and Information Technology (ICCIT): Digital Information Management, Hammamey, 44-47.

[51] Stamatatos, E. (2009). A survey of modern authorship attribution methods. Journal of the American Society for information Science and Technology, 60(3), 538-556.

[52] Tan, R. H. R., and Tsai, F. S. (2010, October). Authorship identification for online text. In Cyberworlds (CW), 2010 International Conference on (pp. 155-162). IEEE.

[53] Zafarani, R., and Liu, H. (2009). Connecting Corresponding Identities across Communities. ICWSM, 9, 354-357.

[54] Liu, S., Wang, S., Zhu, F., Zhang, J., and Krishnan, R. (2014, June). Hydra: Large-scale social identity linkage via heterogeneous behavior modeling. In Proceedings of the 2014 ACM SIGMOD international conference on Management of data (pp. 51-62). ACM.

[55] Goga, O., Loiseau, P., Sommer, R., Teixeira, R., and Gummadi, K. P. (2015, August). On the reliability of profile matching across large online social networks. In Proceedings of the 21th ACM SIGKDD International
Conference on Knowledge Discovery and Data Mining (pp. 1799-1808). ACM.

[56] Jain, P., Kumaraguru, P., and Joshi, A. (2015, August). Other times, other values: leveraging attribute history to link user profiles across online social networks. In Proceedings of the 26th ACM Conference on Hypertext and Social Media (pp. 247-255). ACM. 